\title{
Ophthalmological follow-up of Type 1 (insulin-dependent) diabetic patients after kidney and pancreas transplantation
}

\author{
J. C. Zech, D. Trepsat, M. Gain-Gueugnon, N. Lefrancois, X. Martin and J. M. Dubernard \\ Hôpital Edouart Herriot, Lyon, France
}

Summary. We studied the effect of successful kidney and pancreas transplantation on visual function and diabetic retinopathy in 18 patients with long-term Type 1 (insulin-dependent) diabetes mellitus (17 to 38 years) and with advanced proliferative retinopathy. The average age of the patients was 42 years. Prior to transplantation, 5 eyes were in end-stage ophthalmic complication due to neovascular glaucoma. An ophthalmological follow-up was performed between 1-6 years post-surgery. Analysis of the results showed that the diabetic retinopathy had stabilized after transplantation in 12 cases (66\%) with a supplementary photocoagulation in the majority of cases. The proliferation continued in 4 patients $(22 \%)$ leading to blindness in 2 patients and recurrence of vitreous haemorrhages despite the photocoagulation in the other 2 cases. An improvement was observed on fluorescein angiography in a patient with pre-papillar glial proliferation without photocoagulation. Ten patients were reported to have a cataract and were operated on in two cases before transplantation ; in one patient, the cataract increased following transplantation. In conclusion, the kidney and pancreas transplantation was not effective in our patients in reversing the clinical and angiographic signs of diabetic retinopathy. Moreover, a worsening of the lesions was observed in some cases; this was probably due to the irreversible microangiopathic lesions due to advanced evolution of diabetes.

Key words: Diabetic retinopathy - Pancreas transplantation - Insulin-dependent diabetes mellitus Type 1 diabetes mellitus

Proliferative diabetic retinopathy was rarely observed before the discovery of insulin in 1920 because of the short life expectancy of diabetic patients. Since 1950, each decade has brought progress : anatomo-pathological and retinographic studies, fluorescein angiography and development of photocoagulation, vitrectomy and endoocular surgery, prospective randomised studies and courses of treatment.

Nevertheless, diabetic retinopathy remains one of the main causes of blindness in industrialised countries for patients under 60 years of age.

The difficulty of achieving long-term effective glycaemic control has stimulated research into the development of endocrine-pancreas replacement therapy. At the present time, more than 1500 human pancreas transplantations have been performed worldwide on the hypothesis that if perfect glucose homeostasis could be achieved, the development or progression of the secondary complications affecting the eye and other systems could be prevented or stopped.

The present work deals with ocular findings in 18 patients in whom kidney and pancreas grafts functioned for a minimum of 12 months with comprehensive ophthalmological follow-up.

\section{Subjects and methods}

Patient population and surgical technique. Between September 1983 and May 1989, a total of 18 patients ( 6 women, 12 men) received combined pancreas and kidney transplants with comprehensive ophthalmological evaluation. The average age was 42 years (29-54) and the average duration of diabetes was 26 years (17-38). The average follow-up after transplantation was 37 months (12-72). All the patients presented with proliferative retinopathy before the graft. 18 patients received a combined pancreas and kidney transplantation; the technique for pancreas transplantation being segmental with duct obstruction in 16, total duodenopancreas with bladder drainage in one patient, total duodenopancreas with enteric drainage in one patient.

Patient assessment. All patients were admitted to the ophthalmological centre of our hospital for initial baseline studies 2 months prior to transplantation. Follow-up studies were done six months and twelve months after the date of surgery and subsequently on a yearly basis.

Ophthalmological evaluation consisted of measurement of bestcorrected visual acuity of each eye, a complete eye examination with slit-lamp biomicroscopy, and illustrated and written documentation of retinopathy from biomicroscopy of the posterior 
segment with a Goldmann contact lens, coloured photography and fluorescein angiography of the macular area and specific extramacular regions with suspected neovascularization.

\section{Results}

\section{Visual acuity}

Before surgery, 5 of the 18 patients presented unilateral blindness from neovascular glaucoma ; therefore, we could study 31 eyes (Table 1). After surgery, the visual acuity in the contra-lateral eye of these 5 patients led to blindness in 2 cases, the first by total tractional retinal detachment and the second by evolution to phtisis; the other 3 eyes remained stable. During follow-up, the visual acuity remained unchanged in 24 eyes and improved in 3 eyes. The visual acuity was thus stable or improved in 27 eyes ( 27 out of $31,87 \%$ ). A more detailed analysis showed that the final stable visual acuity was superior or equal to $5 / 10$ for 6 eyes and lower or equal to $1 / 10$ for 10 eyes. In contrast, the visual acuity decreased in 4 eyes $(4$ of $31,13 \%$ ) with evolution to blindness in 2 eyes and to intra-vitreous haemorrhage in 2 other eyes.

Table 1. Characteristics of visual acuity in 36 eyes

\begin{tabular}{ll}
\hline Visual acuity & Number of eyes \\
\hline $\begin{array}{l}\text { Before transplantation } \\
\text { Blindness } \\
\text { (neovascular glaucoma) }\end{array}$ & 5 \\
\hline After transplantation & \\
$\quad$ Stable & 24 \\
$\quad$ Improvement & 3 \\
Decline & 4 \\
Blindness & 2 \\
leading to & 2
\end{tabular}

\section{Cataract}

The development of cataract in each patient was similar (Table 2). Before transplantation, 2 patients ( 4 eyes) had been operated on without intraocular lens, the first by extracapsular extraction and the second by phacoemulsification who had been under medical observation for 5 years. We notice that visual acuity remains stable after the cataract extraction and after the transplantation without the development of either a secondary cataract for the extracapsular extraction or a macular odema for the phacoemulsification. After the graft, 8 patients preserved clear lens and 7 patients with a cataract before the transplantation did not show development of the opacity. In other words, 15 patients out of $18(83 \%)$ remained stable after the transplantation. Only one patient showed development of the cataract with a decrease in the visual acuity of $5 / 10$ to good light perceptions within 6 years. This patient has been operated on 3 times in 18 months following transplantation for reccurence of total tractional retinal detachment and intra-vitreous haemorrhage with cryoapplication, vitrectomy, membrane section and peeling, gas injection and is currently developing phtisis. In this context, the evolution of the cataract is not attributable to pancreas and kidney transplantation. No cataract extraction has been made after the graft.

Table 2. Characteristics of cataract in 18 patients

\begin{tabular}{cc}
\hline Cataract & Number of patients \\
\hline Before transplantation & 2 \\
\hline Cataract extraction & 8 \\
\hline After transplantation & 7 \\
No cataract & 1 \\
Without evolution & With crulution \\
\hline
\end{tabular}

\section{Proliferative retinopathy}

All the patients in this study, undergoing ophthalmological follow-up with regular angiography, presented proliferative retinopathy. Seven patients presented additional odematous retinopathy with alterations such as exsudates, macular odema which remained stable after the graft. Four patients out of 18 presented an increase in proliferation despite photocoagulation, developing to blindness in 2 patients (see above, first by total tractional retinal detachment and secondly by phtisis) and in the other 2 patients leading to intravitreous haemorrhages. Twelve patients stabilized proliferation with photo-coagulation : 7 with photocoagulation before and after transplantation, 5 with photocoagulation before transplantation. Only 2 patients presented a decrease of the pre-papillar neovascularisation on the angiography : first with panretinophotocoagulation before the graft, secondly without photocoagulation for an involutive preretinal gliosis. Thus, proliferation increased in 11 patients but stabilized with photocoagulation after the transplantation in 7 patients. The proliferation stabilized in 5 patients without photocoagulation after transplantation.

Table 3. Characteristics of retinopathy in 18 patients

\begin{tabular}{ll}
\hline Retinopathy & Number of patients \\
\hline $\begin{array}{l}\text { Increase of proliferation } \\
\text { Blindness }\end{array}$ & 4 \\
$\begin{array}{l}\text { (retinal detachment, phtisis) } \\
\text { Intra-vitreous haemorrhage }\end{array}$ & 2 \\
\hline $\begin{array}{l}\text { Stabilization of proliferation } \\
\text { Photocoagulation before and after }\end{array}$ & 7 \\
Photocoagulation before & 5 \\
\hline Decrease of the proliferation & 2 \\
\hline
\end{tabular}


Therefore, we have stabilization or diminution of proliferation in 14 patients who represent $78 \%$. The stable condition of the eyes with advanced retinopathy is presumably due in part to photocoagulation therapy. Twelve patients out of 18 had a panretinophotocoagulation and 5 a focal photocoagulation. Only one patient did not have photocoagulation but presented an involutive preretinal gliosis proliferation with an improvement of the angiography which had been assessed during the previous 6 years.

\section{Discussion}

Visual acuity is a very important criterion for the patients because it influences their independence. We admitted a modification of the visual acuity when it declined or improved by a minimum of 2 lines on the Monoyer's scale. We notice that it is very difficult to appreciate an alteration of the visual acuity when the patient reads $1 / 10$ or when he has only light perception. Visual acuity depends on many parameters and we must interpret the modification in the ophthalmological context (cataract, macular edema, intra-vitreous haemorrhage). Of the 24 eyes with a stabilization of visual acuity, more than $41 \%$ (10 eyes) had a visual acuity lower or equal to $1 / 10$ before and after the transplantation. This is explained by the retinal irreversible alterations due to advanced evolution of diabetes, and it is very important that these patients be able to preserve this small visual acuity for their independence.

Cataract is one of the factors which can influence visual acuity. In our study, the lens is stable after the transplantation for $83 \%$ of the patients without any apparent influence on the medical treatment or on the development of diabetes. With the patients who had a cataract, we verified that the opacity was not an obstacle for angiography and for the retinal photocoagulation in case of an active proliferation after the transplantation. No cataract extractions have been made after the graft. Only one patient presented an increase of the cataract after the transplantation (the other eye was lost because of a neovascular glaucoma) with a fall in the visual acuity and evolution to phtisis following surgery for retinal detachment and intravitreous haemorrhage. In this context, the evolution of the cataract seems to be due to the surgery rather than to the transplantation.

Proliferation was stabilized in $78 \%$ of the patients. We can not allocate this number to the transplantation only because half of these patients necessitated an additional photocoagulation after transplantation. Therefore, the latter patients presented an increase in proliferation after surgery, well stabilized by photocoagulation. Therefore, photocoagulation before the double transplantation pancreas and kidney was not enough to stop proliferation. Four other patients (22\%) presented an incease in proliferation despite the photocoagulation after the graft : this is important because in 2 patients it led to blindness (total tractional retinal detachment and phtisis) and in 2 other patients to intravitreous haemorrhages which prevented any photocoagulation proceedings and diminished chances of stabilizing the proliferation. This may be explained because the patients presented some irreversible retinal lesions secondary to advanced evolution of diabetes (Sutherland et al. 1983; Petersen et al. 1990). Decrease of proliferation rarely occured and has been reported here for 2 patients ; it is difficult to judge the influence of the transplantation on the improvment in these 2 patients.

To conclude, the observations of this prospective study show that proliferation is stable in most of the patients with Type 1 diabetes of long duration after transplantation and subsequent normoglycaemia sometimes complemented by photocoagulation. However, some patients present an increase in proliferation frequently leading to blindness. These results are similar to those of Ramsay et al. (1988) in their study group. It is difficult to make a definite conclusion because of the absence of a control group but we can see in this study that the grafts do not reverse or halt diabetic retinopathy.

\section{References}

Petersen MR, Vine AK, The University Of Michigan Pancreas Transplant Evaluation Commitee (1990) Progression of diabetic retinopathy after pancreas transplantation. Ophthalmology 97:496-502

Ramsay RC, Goetz FC, Sutherland DER, Mauer SM, Robinson LL, Cantrill HL, Knobloch WH, Najarian IS (1988) Progression of diabetic retinopathy after pancreas transplantation for insulindependent diabetes mellitus. N Eng J Med 318:208-214

Sutberland DER, Goetz FC, Ramsay RC, Fryd DS, Najarian IS (1983) Clinical and experimental kidney and pancreas transplantation for diabetes. In: Little HL, Jack RL, Patz A, Forsham PH (Eds) Diabetic retinopathy. Thieme-Stratton inc., New-York, pp 199-221

\author{
Dr. J.C. Zech \\ Hôpital Edouart Herriot, Pavillon C, Place d'Arsonval \\ 69437 Lyon cedex 03 France
}

